\title{
Monitoring of a Hot Melt Coating Process via a Novel Multipoint Near-Infrared Spectrometer
}

\author{
Roland Hohl, ${ }^{1}$ Otto Scheibelhofer, ${ }^{2}$ Elena Stocker, ${ }^{1}$ Sharareh Salar Behzadi, ${ }^{1}$ Detlev Haack, ${ }^{3}$ Kai Koch, ${ }^{4}$ \\ Peter Kerschhaggl, ${ }^{5}$ Dirk Lochmann, ${ }^{6}$ Stephan Sacher, ${ }^{1}$ and Andreas Zimmer ${ }^{7,8}$
}

Received 18 November 2015; accepted 11 February 2016; published online 2 March 2016

\begin{abstract}
The aim of the present work was to develop a PAT strategy for the supervision of hot melt coating processes. Optical fibers were placed at various positions in the process chamber of a fluid bed device. Experiments were performed to determine the most suitable position for in-line process monitoring, taking into account such requirements as a good signal to noise ratio, the mitigation of dead zones, the ability to monitor the product over the entire process, and reproducibility. The experimental evidence suggested that the position at medium fluid bed height, looking towards the center, i.e., normal to particle movement, proved to be the most reliable position. In this study, the advantages of multipoint monitoring are shown, and an in-line-implementation was created. This enabled the real-time supervision of the process, including the fast detection of inhomogeneities and disturbances in the process chamber, and the compensation of sensor malfunction. In addition, a model for estimating the particle size distribution via NIR was successfully created. This ensures that the quality of the product and the endpoint of the coating process can be determined correctly.
\end{abstract}

KEY WORDS: coating; hot melt coating; NIR; PAT.

\section{INTRODUCTION}

Coating of solid dosage forms is an important process step in pharmaceutical manufacturing for various purposes, including taste masking, increasing the shelf life, or tailoring the release profile of the active pharmaceutical ingredients (API) (1-3). In addition, a second API can be delivered to patients by active coatings (i.e., an API is incorporated in the coating) (4). Depending on the purpose of the coating and on the substrate particles (e.g., tablets or pellets), typically pan coating or fluid bed coating are used to apply a thin continuous layer on the substrate. Dependent on the size of the substrate particles, pan coating (widespread used for tablets) or fluid bed coaters (usually used for pellets and mini-tablets) are preferred. Several different implementations of fluid bed

\footnotetext{
$\overline{{ }^{1} \text { Research Center Pharmaceutical Engineering GmbH, Graz, Austria. }}$

${ }^{2}$ Institute for Process and Particle Engineering, Graz University of Technology, Graz, Austria.

${ }^{3}$ Hermes Arzneimittel GmbH, Großhesselohe, Germany.

${ }^{4}$ Romaco Innojet, Steinen, Germany.

${ }^{5}$ EVK DI Kerschhaggl GmbH, Raaba, Austria.

${ }^{6}$ CREMER OLEO GmbH \& Co. KG, Witten, Germany.

${ }^{7}$ Institute of Pharmaceutical Sciences, Department of Pharmaceutical Technology, University of Graz, Graz, Austria.

${ }^{8}$ To whom correspondence should be addressed. (e-mail: andreas.zimmer@uni-graz.at)
}

coaters exist, whereas bottom-spray fluid bed technology is one of the most established methods for pellet coating (2). Typically, an aqueous polymer-containing solution is sprayed onto pellets. However, solvent-free systems have also been developed. For example, hot melt coating (HMC) has been used for many materials, such as paper or textiles, since the early 1940s. In the pharmaceutical industry, hot melt coating has been applied since the 1980s (5). One of the advantages over conventional coating techniques is that no solvent is required. This is because the coating material is applied to a substrate in the molten state, reducing the energy costs associated with evaporation and the (expensive) need for solvent recovery and explosion proofing in case of organic solvents. Moreover, HMC can be performed in modified conventional coating systems (e.g., fluid bed coaters) (5). An excellent understanding and a precise control of the HMC process is required to successfully manufacture and to reduce product variability. The critical process parameters (CPP) are the melt temperature, the air flow rate, the molten lipid spray rate, and the atomizing air pressure (a detailed discussion on the CPPs can be found in Stocker E, SalarBehzadi S, Hohl R, et. al. Applying ICH Q9 quality risk management methods and tools to encourage process development for hot melt coated particles; in preparation). These CPPs significantly affect the critical quality attributes (CQA) of the product, including taste masking. Therefore, the quality of the coating layer is an important and critical parameter for the performance of the drug product. 
In general, the coating quality is characterized by the coating thickness and its uniformity. These parameters should be monitored by sensors providing real-time information about the process state, thus enabling control of the process. Several studies investigated process analytical technology (PAT) applications for the monitoring of coating processes. Most techniques directly rely on the detection of the coating material, including near-infrared spectroscopy (NIRS) (6-9), Raman spectroscopy (10-12), and Terahertz pulsed imaging (13). In a series of papers, we recently reported the use of optical coherence tomography (OCT) as a powerful method for in-line measurement of coating thickness and variability (14-16). Alternatively, the particle growth due to coating can be monitored, e.g., with spatial filter velocimetry (17).

Being non-destructive and fast, NIRS and Raman spectroscopy are established PAT tools for monitoring of coating processes $(6-9,18,19)$. However, the fluorescence of certain coating materials can be a major obstacle with regard to Raman spectroscopy (10-12). Using a correct data pretreatment and reference analytics, successful real-time prediction of coating thickness, uniformity, and drug release via NIRS were reported $(9,18)$. NIRS was also extensively investigated in connection with fast and non-destructive particle size monitoring during granulation processes, typically combined with moisture content determination (20).

All mentioned methods are usually based on point sampling, i.e., the area of measurement is in the range of a few square micrometer (OCT), up to some square millimeter (for infrared). Hence, correct positioning of a probe is critical. This is especially true for processes where spatial gradients exist (e.g., the particle size varies with the fluid bed height).

In the current study, a novel multipoint near-infrared system was used for simultaneous measurements of the coating thickness at various positions in the process chamber of a fluid bed hot melt coater. A single probe may fail or may become useless due to several reasons; very common is the occurrence of fouling. Thus, a multipoint system provides a better monitoring quality and control of the coating process, for example, with regard to coating homogeneity and endpoint control of a process (21-23). Different positions inside the coating vessel were evaluated regarding their susceptibility for fouling and their reliability.

\section{MATERIALS AND METHODS}

\section{Hot Melt Coating}

Hot melt coating was performed in an Innojet ${ }^{\circledR}$ laboratory system Ventilus ${ }^{\circledR}$ V-2.5/1 with an Innojet ${ }^{\circledR}$ Hot-MeltDevice IHD-1 (Romaco Innojet, Steinen Germany). The Ventilus ${ }^{\circledR} \mathrm{V}-2.5 / 1$ is a multipurpose system for granulation, coating, and hot melt coating.

A stable fluidized bed of the substrate crystals is established by the air stream forcing the crystals to follow a helical path inside the process chamber. The process chamber has a volume of 2.51 and is topped by a filter tower. The spray nozzles for the molten coating are situated at the bottom of the process chamber and eject the molten material in a low angle into the process chamber, in all radial directions.

The substrate crystals were provided by Hermes Arzneimittel GmbH, Germany, and were coated with
Compritol ATO 888 (Glattefossé, Nanterre Cedex, France). The coating process was performed for $30 \mathrm{~min}$ at a constant product temperature of $55^{\circ} \mathrm{C}$. Atomizing air pressure was set to 1 bar. These settings were applied in all experiments.

\section{Near-Infrared Spectroscopy and Probe Positioning}

A new spectrometer prototype, Helios EyeC Multifiber, provided by EVK (EVK DI Kerschhaggl GmbH, Raaba, Austria) was used to monitor the process. The spectrometer is based on available hyperspectral imaging systems. The MCT-sensor chip (mercury-cadmium-telluride) allows effective detection of a spectral range in the near-infrared region of 1000-2250 nm. A special optical unit maps up to 25 probes, which can be attached via typical SMA905-ports to the spectrometer, to the entrance slit of the imaging system. Thus, this enables the simultaneous acquisition of all probes. With this setup, acquisition of spectra can be completed very quickly, and spectral drifts between several probes do not occur.

In each coating run, measurements were performed simultaneously at three positions. One sensor was permanently fixed at level 2 and looking in a radial direction during all experiments. This sensor was defined as the main sensor. The other sensors were placed at different levels, as shown in Fig. 1. These sensors are termed further control sensors.

The three probes used consisted of bifurcated fibers, capped with a sapphire window (Ocean Optics Inc., Dunedin, USA). They were mounted inside the process chamber at different positions and were pointed in different directions, as shown in Fig. 1. The height levels of the sensor positions are marked 1 to 3 . The directions of the sensors were chosen to be in circumferential direction (C), in the upward (U) direction, downwards-looking (D) onto the fluid bed, and in a radial (R) direction, i.e., from the vessel rim towards the center of the fluid bed.

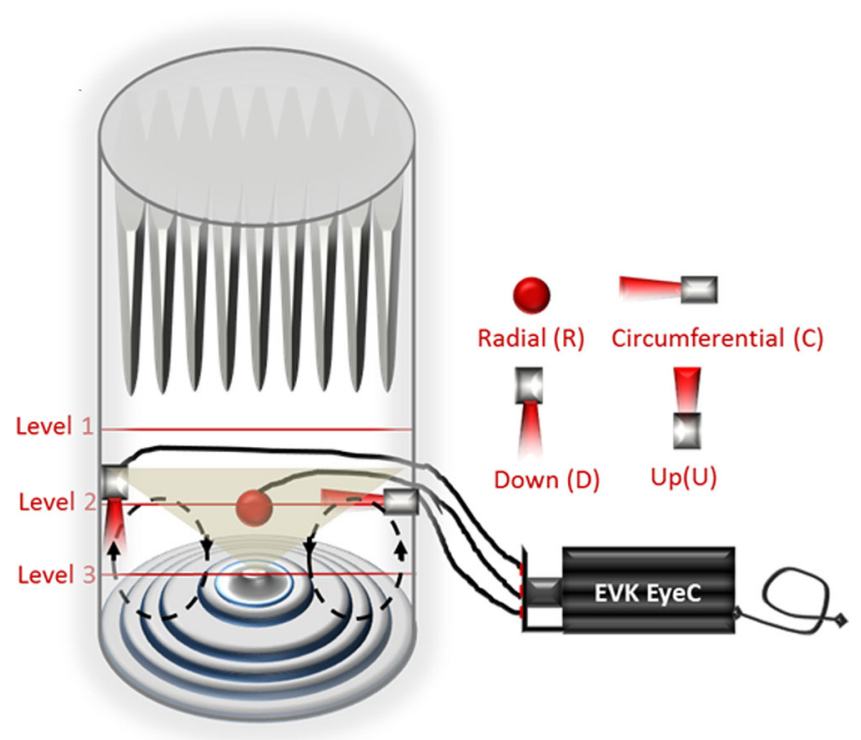

Fig. 1. Fluid bed vessel with a bottom-spray nozzle and EVK system Helios EyeC. NIR optical fiber probes were turned in different directions: $C$ circumferential, $U$ upwards, $D$ downwards, and $R$ in radial direction towards the center 
The spectra were acquired with an integration time of $1500 \mu \mathrm{s}$ and a frequency of $105 \mathrm{~Hz}$. After every $5.3 \mathrm{~s}$, the recording was interrupted for about $8 \mathrm{~s}$ to flush the working memory, resulting in about 60,000 spectra obtained during the process time of half an hour. The interpretation of spectra was performed with MATLAB R2011b (version 7.13.0.546, TheMathWorks Inc., Natick, Massachusetts, USA) and using the Statistical Toolbox, for off-line analysis as well as SIMCA and Simca-online, respectively (SIMCA version 13.02, SIMCA-online version 13.01, Umetrics, Umeå, Sweden) for in-line analysis. Since all spectra were recorded by one single CCD chip of the EyeC system, typical setbacks associated with multiple spectrometers (e.g., the need for individual models, time delays, differences in the resolution) were eliminated.

Right before the coating process was started, white and dark reference spectra were recorded; for creating the dark reference, the sensors were disconnected from the spectrometer, and for the white reference, Spectralon ${ }^{\circledR}$ (Labsphere, North Sutton, $\mathrm{NH}$ ) was used at a distance of $2 \mathrm{~mm}$ from the probes. The direction and position of the main sensor $(\mathrm{R})$ was not changed, during the experiments. The control sensors were used at different height levels and different directions. Many positions proved to be in vain, due to having a too low particle density, or not enough movement in front of the sensor, to give reliable results. Three experiments will be discussed in detail in this manuscript, which are listed in Table I.

\section{Compensating for Window Fouling}

Another crucial issue associated with HMC is window fouling, which can render the sensors ineffective. For example, the endpoint can be detected too early due to coating material deposited on the sensor head. Window fouling occurs when the molten lipid recrystallizes on the capping of the NIR fibers, rather than on the particles in the process chamber, or when abrasion of the coated particles takes place. As such, window fouling can be different at different positions. To compensate for window fouling, extended multiplicative signal correction can be used (EMSC) (24). As proposed in (25), the collected spectra were separated according to:

$$
A_{\text {measured }}=a \cdot A_{1}+b \cdot A_{\text {crystal }}+c \cdot A_{\text {coating }}+d \cdot A_{\lambda}+e \cdot A_{\lambda}^{2}+R
$$

The parameters $a, b, c, d$, and $e$ are calculated for every time step but are constant over the considered spectral region. $A_{1}$ is a constant value across all wavelengths, which mainly reflects the influence of the crystal distance. $A_{\text {crystal }}$ is the characteristic spectrum (taken off-line) of pure substrate crystals. Therefore, if crystals are near the probe window and give a notable absorption spectrum, $\mathrm{b}$ is high. Since $a$ and $b$ depend on the crystal distance, they show a correlation. $A_{\text {coating de- }}$ scribes spectral content attributed to coating. It was obtained by taking off-line spectra of the samples collected during the coating process. $A_{\lambda}$ and $A_{\lambda}{ }^{2}$ represent linear baseline offset and quadratic curvature.

By taking into account the existing correlations between $a, b$, and $c$, i.e., the geometrical-based relation of crystal distance and crystal and coating proportion in the spectra, it is possible to distinguish between the coating, adhered to crystals, and coating adhered to the probe window (25). Thus, the increase of coating on the crystals and on the NIR probes can be followed separately. The obtained corrected EMSC coefficient is then directly proportional to the detected coating mass.

This calculation is performed for every time step (i.e., with $105 \mathrm{~Hz}$ ). To avoid cluttering, in the following figures, the mean value over $0.57 \mathrm{~s}$ and the corresponding standard deviations over the same time period are indicated. Numerical values are given relative to the coating target value.

\section{Off-Line Measurement of the Particle Size Distribution}

Particle size distribution was measured using the QICPIC dynamic picture analysis instrument with the RODOS dry dispersion unit and the VIBRI vibrating conveyor from Sympatec (Sympatec GmbH, Clausthal-Zellerfeld, Germany). QICPIC is a high-speed camera with a stroboscopic light source, which can take up to 450 pictures per second of dispersed particles moving through the measurement zone. The associated software WINDOX 5.6.0.0 calculates the PSD and shape factor distributions of the measured particles by evaluating their projected area in the pictures. The particle diameters $\mathrm{x}_{10}, \mathrm{x}_{50}$, and $\mathrm{x}_{90}$ were determined for the particle volume distributions.

\section{Coating Prediction via PLS}

The calibration of the NIR setup was performed off-line, using samples taken every 5 min during a coating process. Samples were distributed homogeneously on a rotating table (Sensor Turn, WLP-TEC GmbH, Göttingen, Germany). The table rotated to mimic particles passing the probe and to avoid subsampling. To collect the calibration spectra, the three fiber optic probes were fixed on an acrylic glass disc mounted $2 \mathrm{~mm}$ above the rotating material. For the in-line measurements, it was also necessary to develop a PLS (projection to latent space) model for the prediction of the coating mass. The model is similar to the one presented in (26). The model was developed by recording several batches during the entire process time. Afterwards, only those batches which performed successfully (as determined afterwards by drawing samples and analyzing them off-line) were considered for inclusion in the model. Off-line-analysis took place via

Table I. Positions and Directions of the Near-Infrared (NIR) Fibers for Different Experiments of the Hot Melt Coating Process, Listed in this Article

\begin{tabular}{llll}
\hline & Main sensor & Control sensor 1 & Control sensor 2 \\
\hline Experiment 1 & Level 2 (radial direction) & Level 2 (downwards-looking) \\
Experiment 2 & Level 2 (R) (radial direction) & Level 1 (downwards-looking) & Level 2 (circumferential direction) \\
Experiment 3 & Level 2 (R) (radial direction) & Level 3 (upward-looking) & Level 2 (circumferential direction) \\
\hline
\end{tabular}


dynamic picture analysis, as described in "Off-Line Measurement of the Particle Size Distribution" section. It was assumed that the increase in particle diameter over process time can be attributed to the growth of the coating layer on the particles. This was supported by microscopic images (taken with a Leica DM 4000 equipped with a Leica DFC 290 camera), which allowed optical distinguishing of the coating layer from the substrate material. The score plot for a single calibration batch is shown in Fig. 2.

As target variable, the batch maturity, i.e., the normalized process time, is used. Thus, the endpoint of a successfully coated batch is 1 . More details on such batch models can be found in literature $(27,28)$. SNV normalized spectra over nearly the whole spectral range (1100-1800 nm) were used for the prediction of coating. Three PLS components were used, resulting in a model with a RMSECV of 0.033 (in units of normalized time, i.e., root mean square error for predicting the batch maturity of the cross-validation batches is approximately $1.5 \mathrm{~min}$ ). The regression vectors indicate that the increase of coating material, and the decrease of substrate material, in the obtained spectra are the variables relied on.

As this model only provides a regression vector for the obtained spectra (i.e., is sensitive to the increase of coating material in the obtained spectrum), the validity of this model can only be guaranteed for one position and reproducible fouling over several batches (as window fouling is intrinsically calibrated into the model). Hence, the model can only be applied, when the correction in "Compensating for Window Fouling" section has shown that fouling occurs as expected.

\section{Setup for In-line Monitoring}

An FPGA (field programmable gate array) chip housed in the spectrometer is used for control of the spectrometer. In principal, the FPGA can be used to perform calculations on the acquired spectra at high speed (29). In the current application, however, no further steps are performed on the FPGA, but the raw detected intensity is exported. The multipoint spectrometer was set to an automatic mode for in-line-monitoring, to regularly acquire and save the detected intensity data into files. This has the additional advantage that the original spectral data is preserved (and thus archived) as demanded by the regulators.

In an intermediate step, the folder containing the spectral data is supervised remotely and newly written files are loaded and processed further in an in-house developed program. The first step is the conversion of the recently acquired intensity $I(t)$ to absorbance, by including the previously determined white $I_{\text {white }}$ and dark $I_{\text {dark }}$ reference, via

$$
A(t)=-\log _{10}\left(\frac{I(t)-I_{\mathrm{dark}}}{I_{\text {white }}-I_{\mathrm{dark}}}\right) .
$$

The absorbance spectra are then used, as described in "Compensating for Window Fouling" section to correct for window fouling, for off-line analytics. A mean absorbance over $5 \mathrm{~s}$ is calculated for in-line supervision. These values are then written to an OPC server, running on the same machine, as illustrated in Fig. 3. OPC (Object Linking and Embedding for Process Control) is a standardized software interface for the exchange of plant data between instruments and controllers of different manufacturers.

A third computer serves as the final supervision station. Here, the data are imported regularly from the OPC server via two different APIs (application programming interfaces) that serve as OPC clients, namely the Batch Table Wrapper API and OPC SIM API. The communication between OPC server and clients on different PCs is a delicate task, regarding communication protocols and data safety; details are omitted here. The data are then used in a real-time prediction system (i.e., SIMCA-online). This system performs chemometric analysis

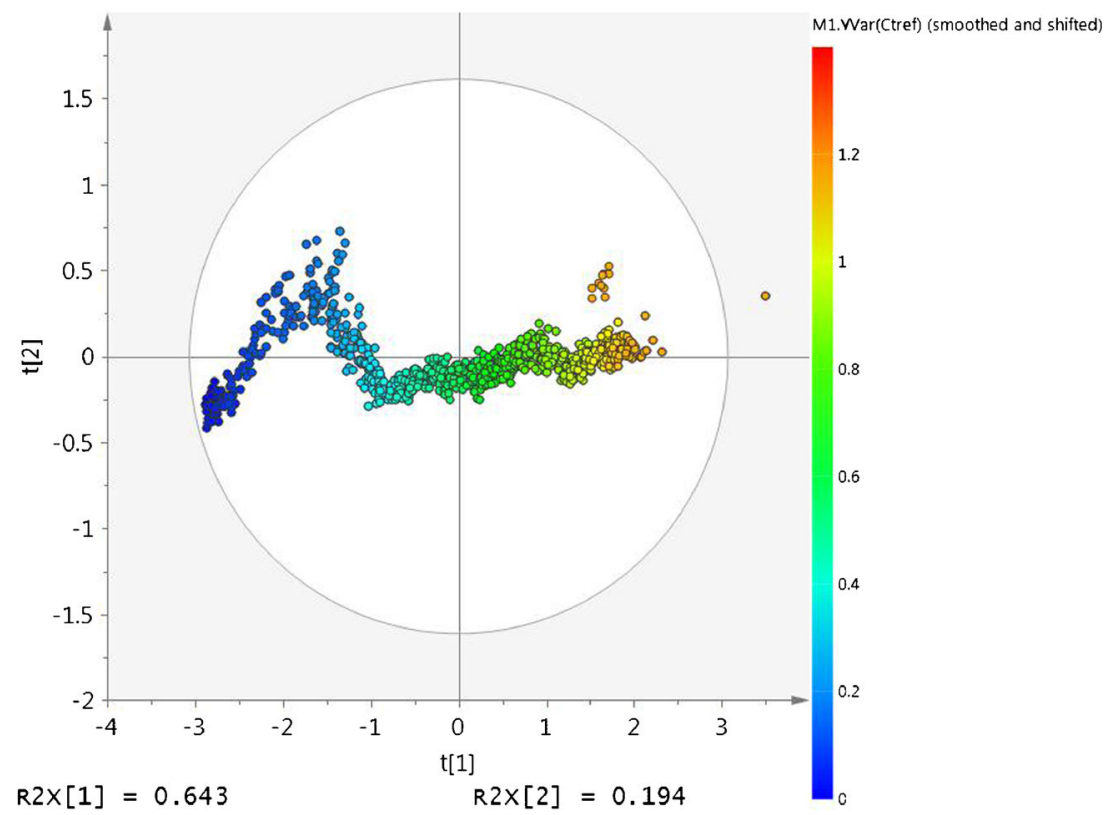

Fig. 2. Score scatter plot of a calibration batch for the PLS model. The color indicates time. Hence, it can be seen that the HMC processes wander from left to right, until they are finished. Whereas the first component nicely increases with time, the second component has to compensate for some fluctuations. This may be due to a badly controlled coating feed 


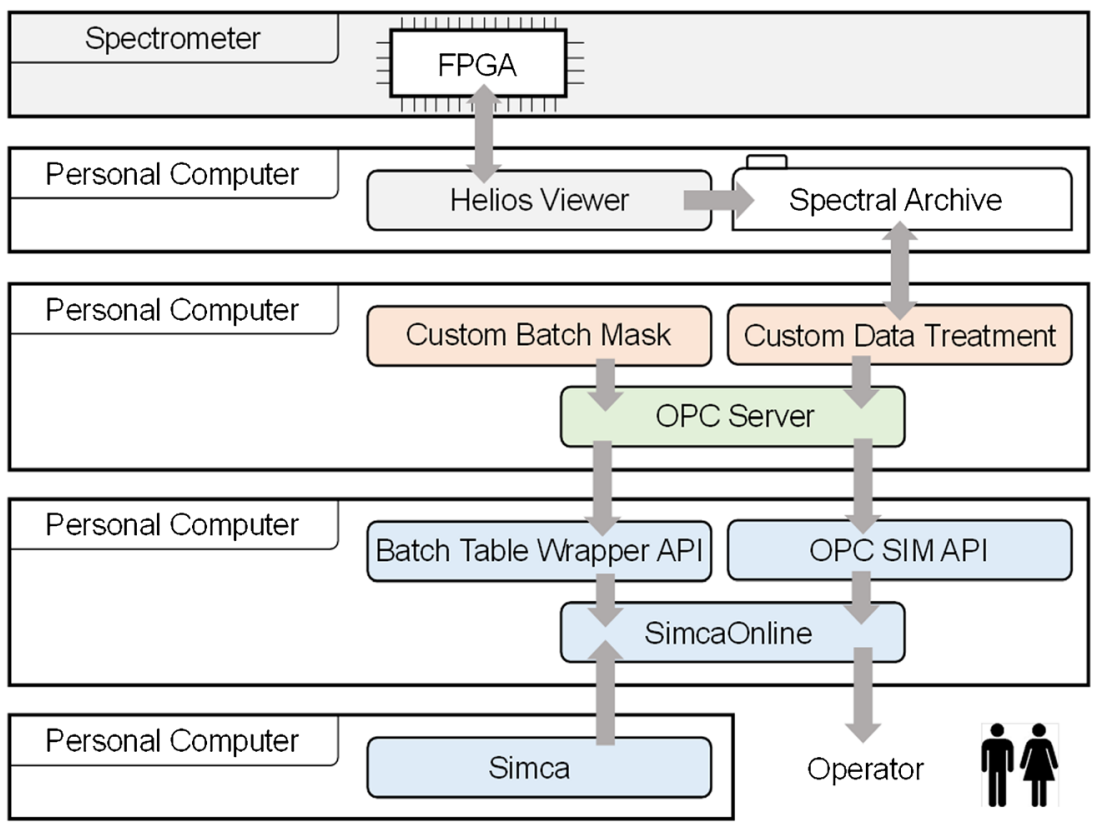

Fig. 3. Schematic overview of the data flow. Gray fields are performed on the spectrometer and the accompanying computer. Orange fields represent our custom program, used for entering batch data and handling of spectra, respectively. Green marks the OPC server (MatrikonOPC, Köln, Germany). Blue fields are products from the company Umetrics AB. APIs are used by SIMCA-online for communicating with the OPC server and administrating the batch data. SIMCA itself is used to prepare the chemometric model

of the data, based on the chemometric model, which was established beforehand, providing a status report and possible warnings to the user.

\section{RESULTS}

\section{NIRS Measurements}

Experiments were performed with sensor positions given in Table I. The applied coating is shown for all experiments as ratio to a target coating level. Hence, a coating ratio level of 0 represents the lack of coating and a value of 1 roughly a coating thickness of $75 \mu \mathrm{m}$. A usual coating process should end at a range of 1 . In Fig. 4, the experiment 1 according to Table I is shown; the higher coating ratio reported by the sensors is caused by window fouling of the sensors during the process time.

In Fig. 5, the same process data are presented, now with the correction for window fouling. As can be seen, the NIRmeasured coating ratio decreased and a final value of approximately 1 was reached, which is in agreement with the off-line samples; the off-line calibration samples are within the standard deviation of the in-line measurement. Therefore, by using the off-line calibration and without applying the presented signal correction, the process would potentially be stopped too early before reaching the final coating ratio.

The results of experiment 2 are shown in Fig. 6. In this experiment, control sensor 1 was fixed at an unsuitable position above the fluid bed at level 1 . This sensor was not able to see the product adequately because the product stream was too far away, and only window fouling was detected. In contrast, the main sensor and control sensor 2 were mounted at level 2. The same increase of the coating ratio can be observed at both positions, indicating that particles are coated homogeneously.
Additionally, a rough spatial assignment and a detection of disturbances are possible by considering the different sensor positions and directions, as shown in Fig. 7. The disturbances detected by the main sensor and the control sensor 2 might be caused by substrate crystals falling down from the filter. Control sensor 1 shows no disturbance at this specific process time. This may be due to the fact that this sensor was looking downwards from top to bottom, and the disturbance was rather short and local in the fluid bed.

In the experiment 3 , according to Table I, shown in Fig. 8, control sensor 1 was fixed at level 3 , at the bottom of the process chamber, and control sensor 2 above the fluid bed at level 1 . The control sensor 1 was not able to follow the coating because in front of the sensor a dead zone was created where no particle movement occurred. Control sensor 2 was not able to see the product because it was too distant. The process could only be followed with the main sensor, as it was mounted at level 2.

All other possible positions for control sensor 1 and control sensor 2 were tested. The best position for the sensors is level 2 in the process chamber. At this level, the process could be followed with all three sensors. The sensors were mounted at level 2 for all following experiments.

\section{In-Line Prediction of Particle Size Distribution}

The reference measurements were generated by drawing samples and measuring them with NIR as described in "NearInfrared Spectroscopy and Probe Positioning" section, followed by QicPic measurements for several batches. As can be seen in Fig. 9, the predictions are successful, yet scatter wildly. The color is according to the DModX value (30), indicating that some of the measurements differ in their spectral response. 

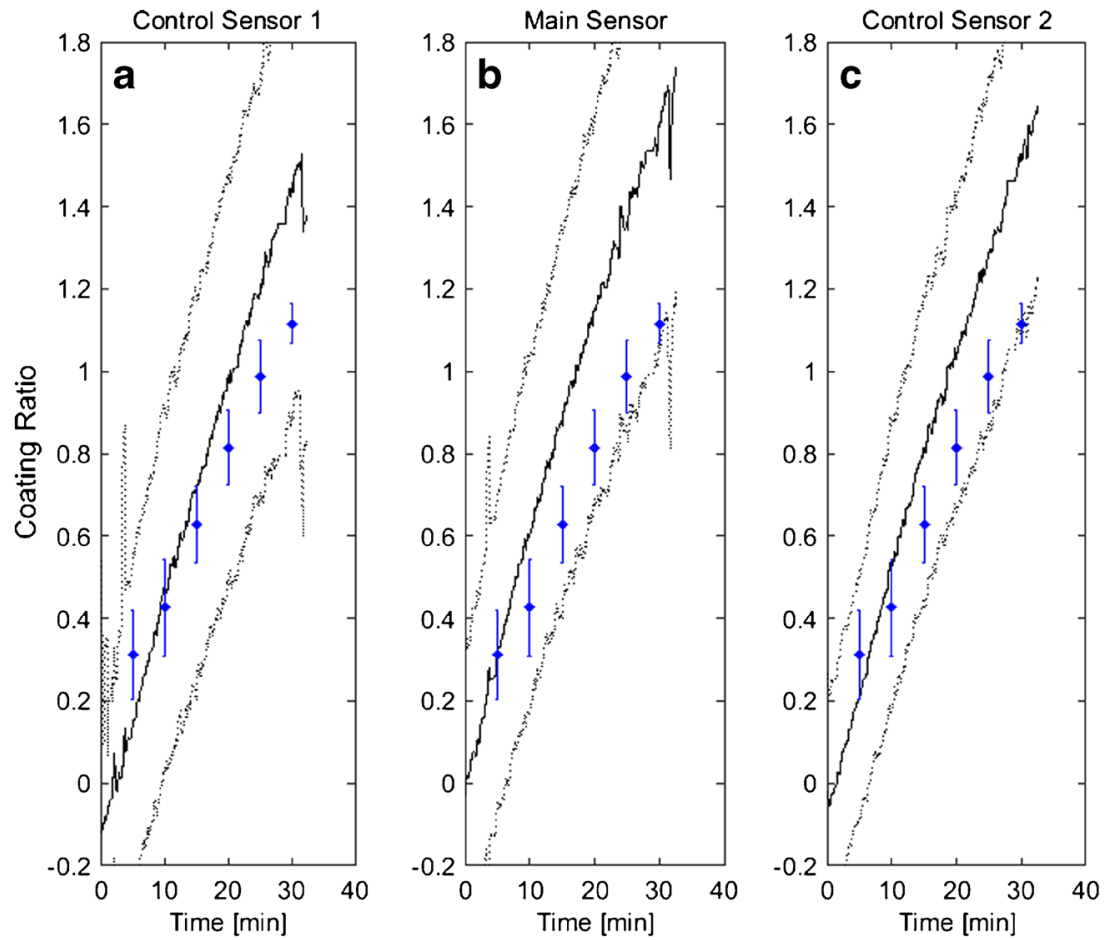

Fig. 4. a-c Experiment 1 according to Table I, coating ratio vs. time without correcting for window fouling (solid line). The dotted line is the standard deviation of the coating ratio. Blue dots represent the off-line measured values. As can be seen, the off-line measurement shows a lower coating ratio. The higher coating ratio reported by the sensors is caused by window fouling of the sensors during the process time

The PLS model is based on the second derivative of the spectral range between 1375 and $1650 \mathrm{~nm}$. Three PLS components are used; taking a look at the PLS weights, it is clear that the first component reflects the diminishing of the substrate material during the coating process, whereas the second and third component represent contributions of the peak
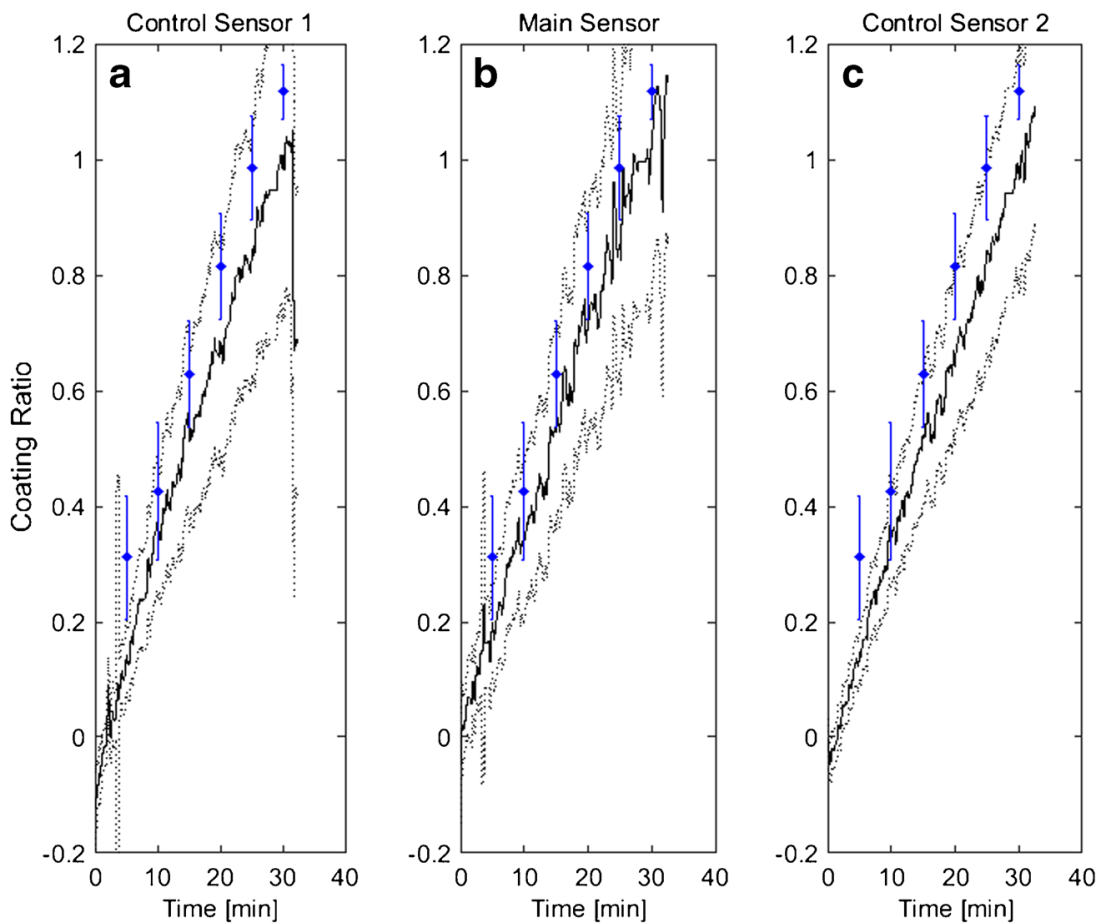

Fig. 5. a-c Experiment 1 according to Table I, coating ratio $v$ s. time after correcting for window fouling. The dotted line is the standard deviation of the coating ratio. Blue dots represent the off-line measured values. At minute 34, the process was stopped, and depending on the direction of the probes, their ability to still see particles in the settled bed is different, hence the different values of coating ratio at the very end 

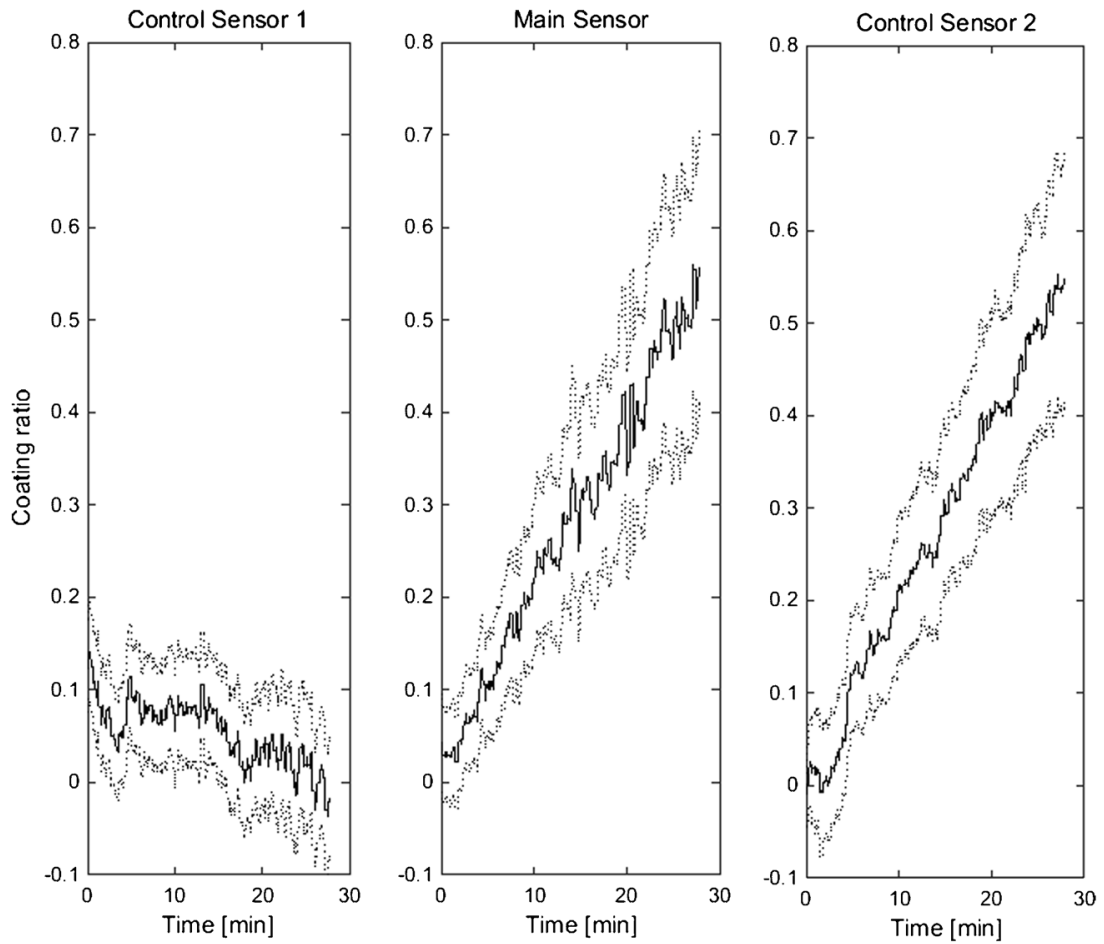

Fig. 6. Experiment 2 according to Table I, coating ratio $v s$. time for experiment 2 . The solid lines represents coating ratio and the dotted lines are the standard deviations. The sensor in the left chart was mounted at an unsuitable position

flanks. Hence, the particle sizes were determined, based on chemical information, which is insensitive to variations of the particle-sensor distance. The cross-validation groups are distinct batches. The obtained root mean square error for cross- validation (RMSECV) for the prediction of the $\mathrm{x}_{50}$ parameter was below $30 \mu \mathrm{m}$. The predicted values during the process (with NIRS) and reference values (with QicPic) for the calibration measurements can be seen in Fig. 10.
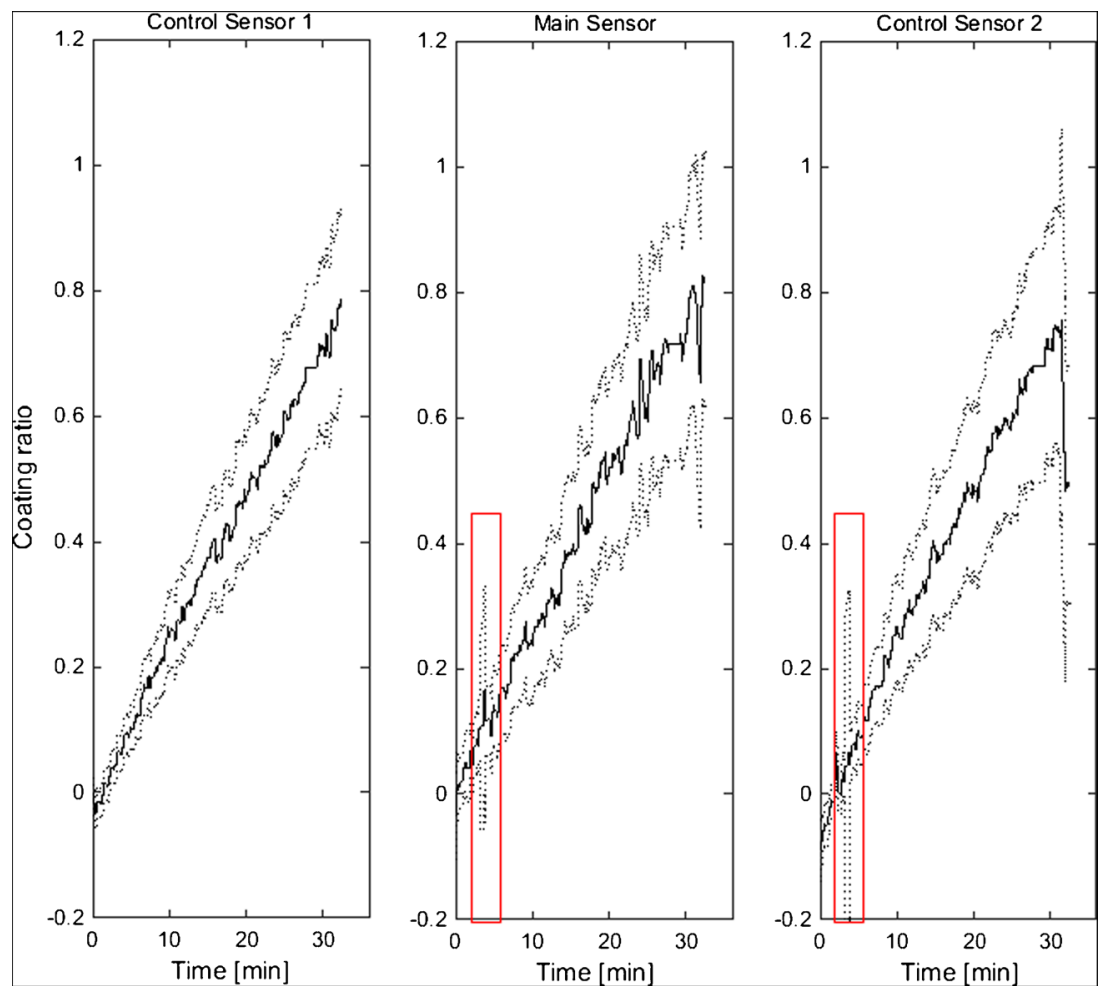

Fig. 7. Coating ratio recorded by the three sensors over time. The black lines are the measured-to-desired coating ratio; the black dotted lines are the standard deviation of the coating ratio. The main sensor and the control sensor 2 detect a process upset at the same time, marked with a red rectangle 

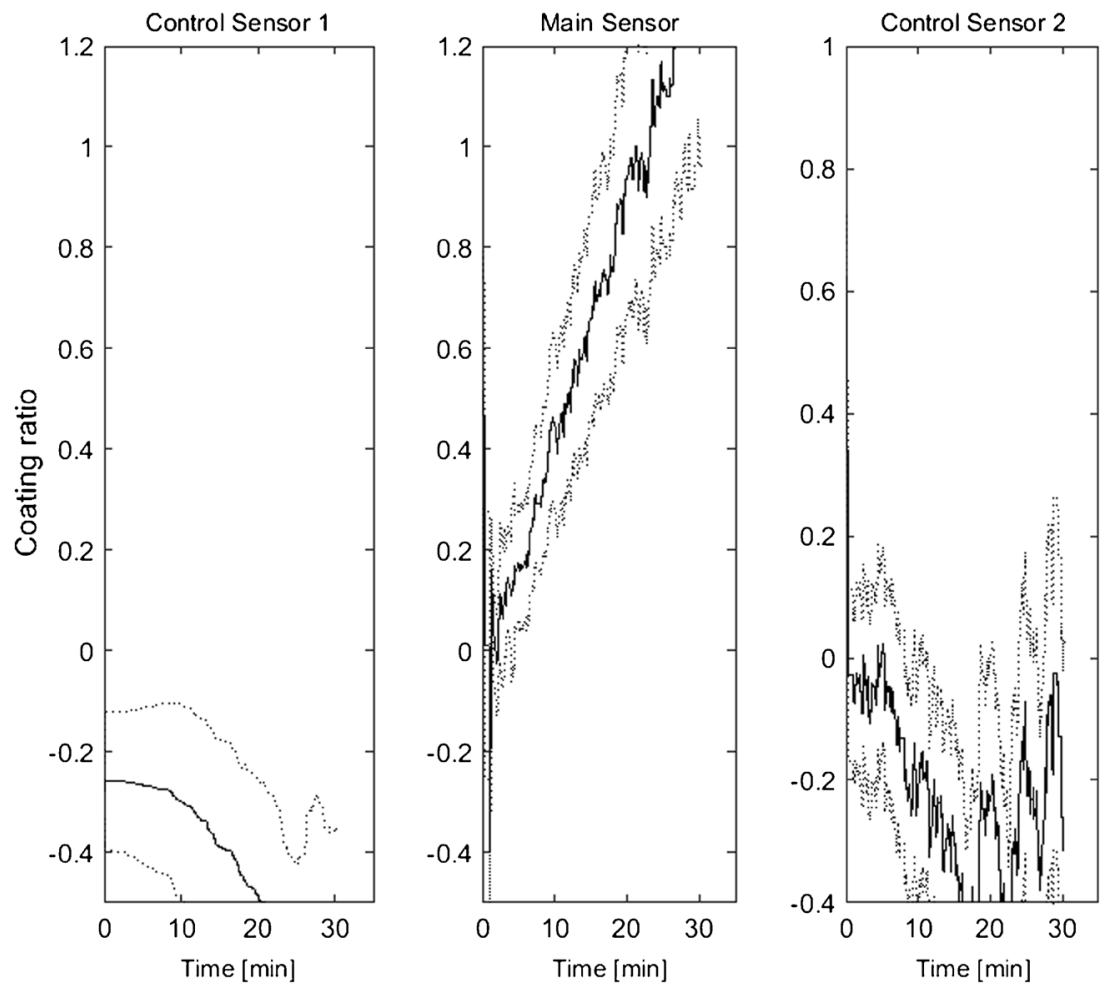

Fig. 8. Experiment 3 according to Table I, coating level recorded by the three sensors over time. The solid lines are the coating ratio; the dotted lines are the standard deviation. Both control sensors (left and right graph) fail, due to their unsuitable positioning

Additionally, different size parameters (i.e., $\mathrm{x}_{10}$ and $\mathrm{x}_{90}$ ) can be modeled the same way. However, as those share the same calibration samples, the relationship in the predictions between different particle size parameters is nearly linear.

In Fig. 11, the predicted particle diameter is shown over process time. Hence, it is possible to follow the particle size over time. Additionally, it can be seen, that the obtained particle size slightly varies depending on the sensor position. In this experiment, the probes were arranged in the same configuration as experiment 1 ; and thus, it can be concluded that the particles are slightly separated inside the fluid bed.

\section{In-Line Monitoring of the Hot Melt Coating Process}

The crucial step for enabling in-line control is the realtime implementation of the analysis-hitherto performed offline-into an in-line system. The spectrometer represents the data in a hypercube, which incorporates the obtained intensity values, dependent on position, wavelength, and time. Hence, a second set of measurements results in a cube with dimensions of $25 \times 256 \times 105$ (25 channels, not all are used; 256 distinct wavelengths, and an acquisition rate of $105 \mathrm{~Hz}$ ).

Whenever those values are written as a complete file, into the supervised folder by the spectrometer software, the files are read again using our custom program. The three used measured positions are extracted from the 25 channels. Additionally, the program performs the necessary spectroscopic steps, i.e., conversion of intensity to absorbance by including background spectra. Furthermore, a mean of 525 spectra over a time step of $5 \mathrm{~s}$ is taken. These data are then written by an OPC client on the defined OPC server.

Hence, a number of $3 \times 256$ spectral variables are present on the OPC server. Additionally, some control variables are stored on the OPC server (e.g., batch information as batch number and start time, timestamps of the spectra, control variables that the spectrometer is online, and the custom program is running OK, etc.). The data are persistent on the OPC server, hence available whenever the data are questioned, and only replaced by newer data.

The data are then read by SIMCA-online, in a regular interval of $5 \mathrm{~s}$ (chosen in our setup). The data are consecutively interpreted by a chemometric model, developed in SIMCA beforehand. Thus, selection of spectral bands and projection into an existing PLS model are performed via SIMCA-online.

In a final step, an overall maturity parameter, indicating the process state via a single variable, is created via hierarchical modeling. The already described PLS model for coating thickness prediction serves as bottom model. The obtained scores in these models, for the first two latent variables and for every channel, thus six variables, serve as input for a top model. This top model is again a PLS model and is regressed towards a maturity parameter ranging from 0 at process start to 1 for achieving the desired coating thickness at all three channels. That means, the large original data set has been condensed down into a single variable, indicating the progress of the hot melt coating process, which can be interpreted by operators, and serve as decision indicator.

Finally, it is necessary to define limits for (1) determining a successful process end and (2) deciding if a process is out of specification. When determining a successful process end, the predicted coating level of all three positions must be within 1 to 1.1 of the desired coating level, at all three positions at the same time, and no other alarm must have occurred. The limits for detecting an out-of-bounds process, or confirming a still successful process, are the following: 

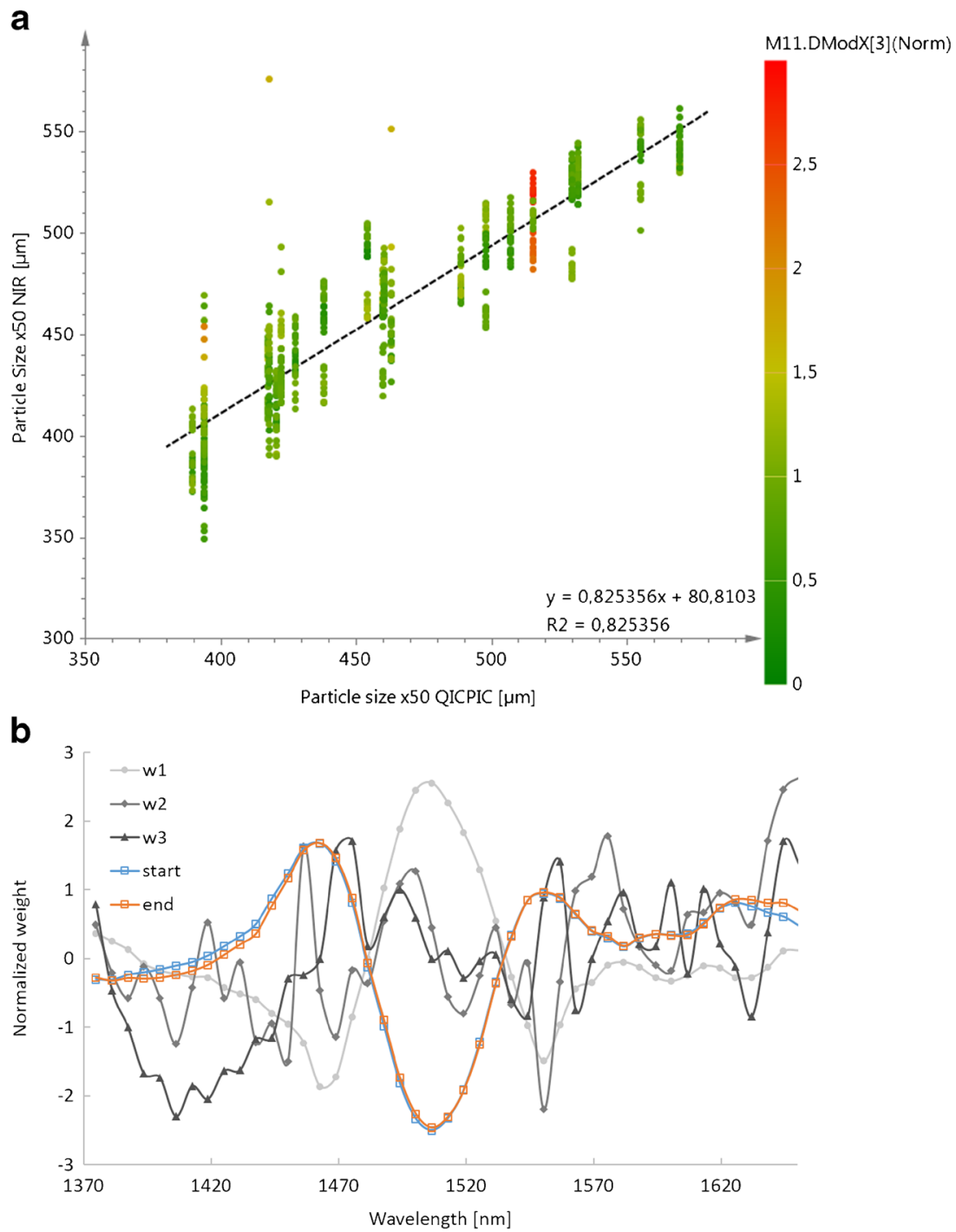

Fig. 9. a, b Top predicted and reference values for NIR and particle size parameter $\mathrm{x}_{50}$ obtained by QicPic. Bottom the three PLS weights for the first three components are shown (w1, w2, w3). Additionally, the second derivative spectra from process start and end are depicted. Note, that those have been scaled, in order to make them comparable

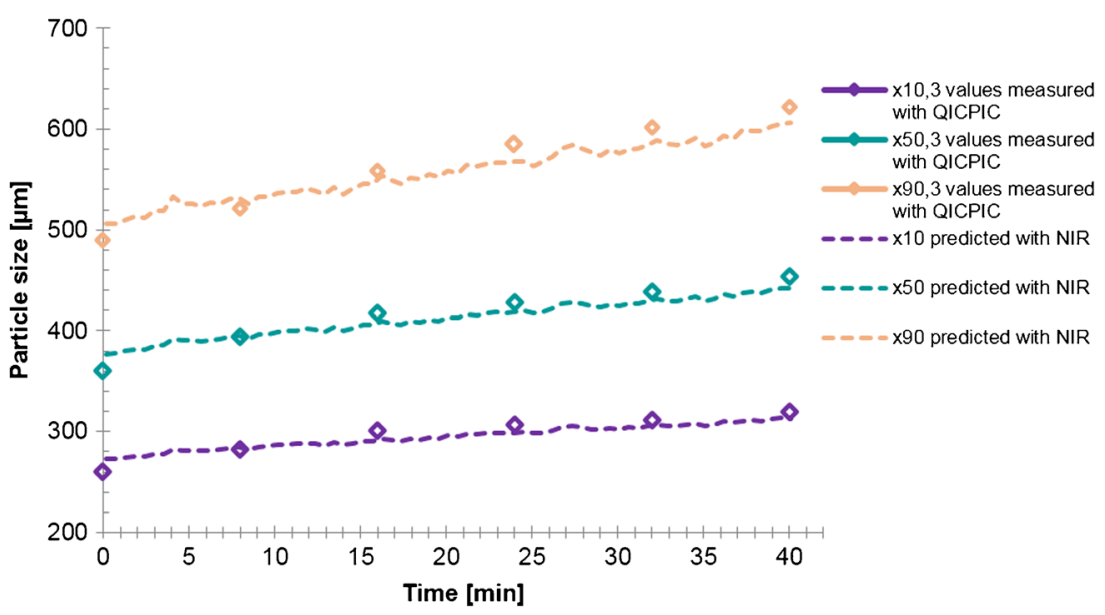

Fig. 10. Predicted value and reference value for the calibration measurement of the particle size $\mathrm{x}_{50}$ 


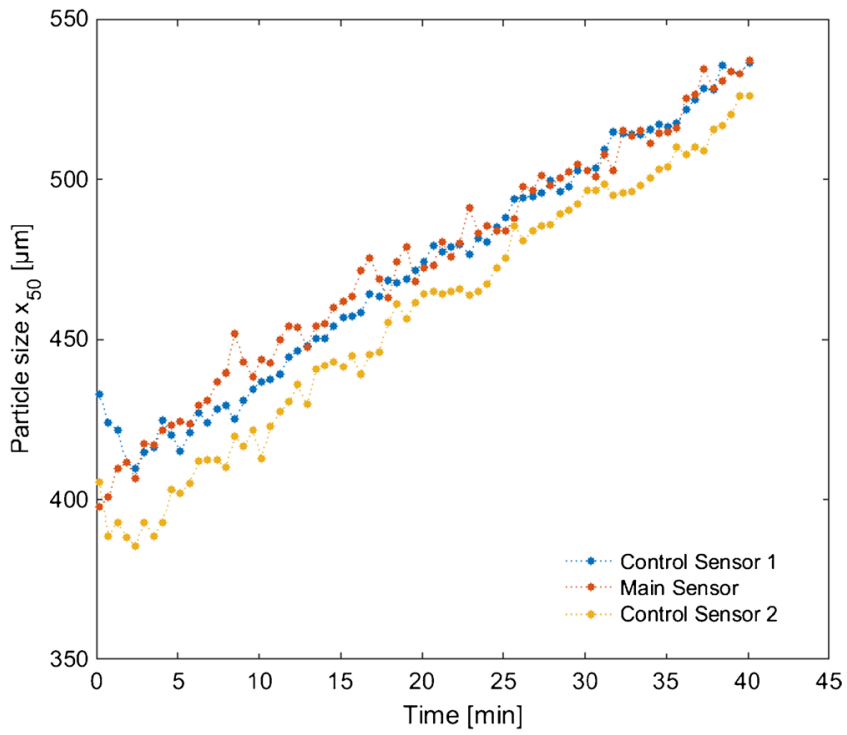

Fig. 11. Prediction of the particle size during the coating process. Evidently, the particle size increases as the particles gain coating mass over time. However, different positions give slightly different results. The sensors were mounted according to experiment 1

- A confidence limit for the desirability variable was established by previous batches. As this variable contains all three probes, a deviation in any probe will lead to deviation in this variable.

- Hard alarm limits were set in the predicted coating amount for all three channels, as well as on the score values of the individual measurement positions.
- The DModX value (i.e., the residual between the reconstructed spectra in the PLS model and the actual measured spectra) is supervised. Here, a large value indicates that the obtained spectra have a different structure then expected, and investigation is needed.

A process monitored with SIMCA-online, showing the indicator variables over time, can be seen in the screenshot in Fig. 12. Problems appeared as sometimes only a portion of a spectrum was transmitted, before the reading process started, and thus was mixed with the previous spectrum. These piecedtogether spectra led to a high DModX value and could not be interpreted correctly. This problem should be avoided with new software versions, as a spectrum is treated as a vector and not an array of independent numbers anymore.

\section{DISCUSSION}

\section{Probe Positioning and Multiple Probes}

The examples shown in "NIRS measurements" section highlight the fact that the sensor positions must be chosen carefully. If only one sensor is used, and its position is not chosen carefully, in-line monitoring will ultimately fail.

The sensor performance at different levels provides a judgment on the different positions. As expected, the central level (level 2) is feasible for process monitoring. Regarding the lower level (level 3), the problem that the sensor was completely covered by crystals arose (although not in all experiments). This also happened once at level 2 for an uplooking (U) sensor. Hence, one must be careful not only to

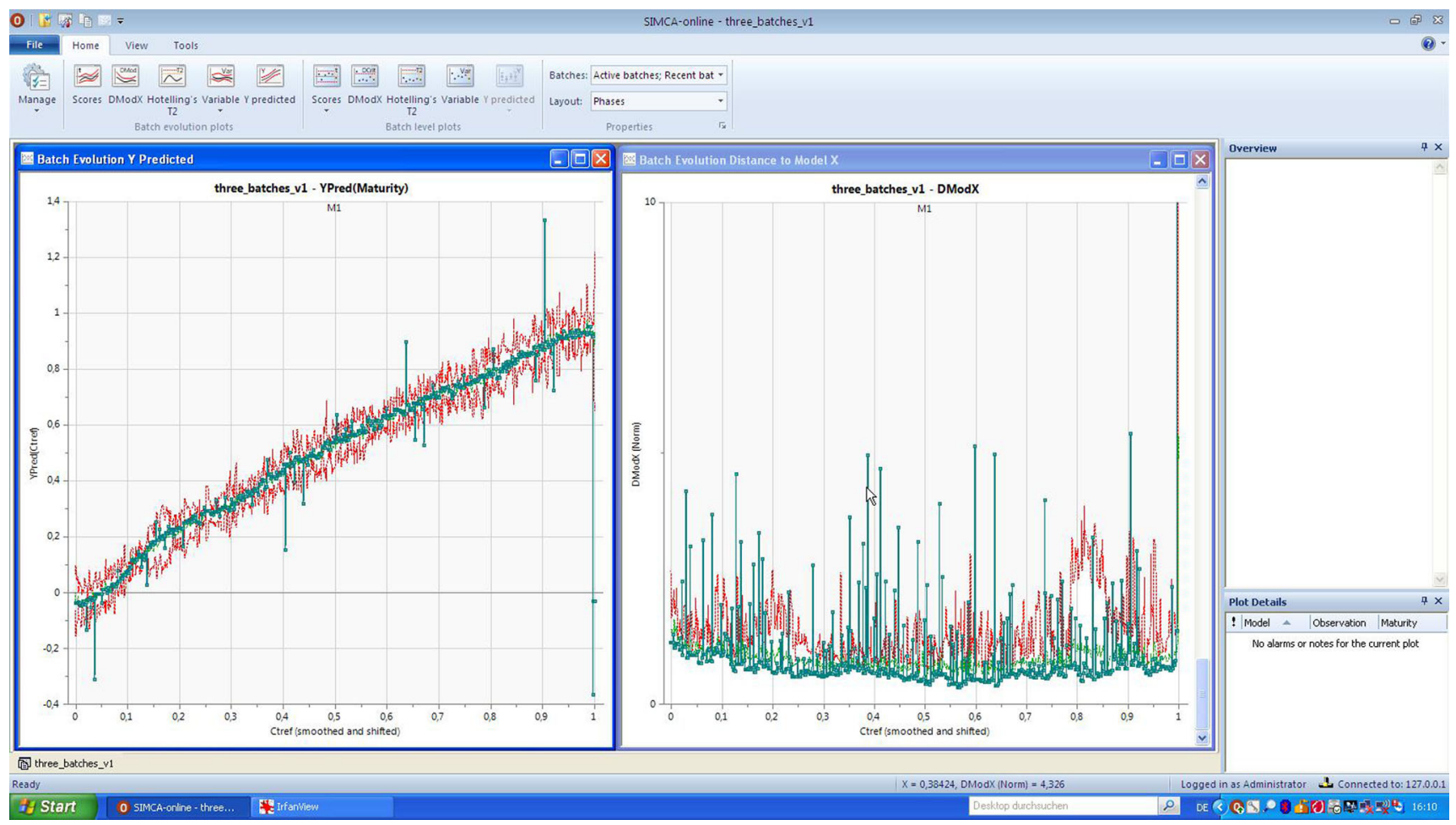

Fig. 12. Screenshot of a hot melt coating process monitored with SIMCA-online. On the left-hand side, the running maturity variable and the allowed corridor are shown. On the right-hand side, the DModX variable is plotted. It can be noticed that the process is running smoothly, but singular outliers appeared. An investigation unveiled, that those outliers were caused not by real measured events but by incompletely transferred spectra to the OPC server 
avoid fouling of the sensors by coating but also the substrate crystals themselves can clog the sensor if the position is unsuitable. Both problems occur individually and in combination, when positioning the sensor at the lowest level (level 3). However, sometimes these positions worked remarkably well. On most occasions, the number of crystals observed was too few to provide reliable results. This indicates that the effective height of the fluid bed varies from process to process (although the experimental settings are the same). This may be caused by environmental variables, changes in the raw material properties, and other factors. Consequently, it also shows that a single experiment is not reliable in deciding for or against suitable measurement positions in fluidized systems.

Through using a multiple-sensor approach, process disturbances, such as those shown in Fig. 6, can be detected and corrective actions may be initiated, bringing the process back into the desired operating space. Moreover, malfunction of one sensor can be compensated. With regard to a hot melt coating process, the corrective actions could, for example, be an increase/decrease of process time and spray rate.

Figure 13 (sensor positions according to Table I, Experiment 1 ) shows the coating ratio for the three sensors. It can be observed that control sensor 1 follows the trajectory of the coating process until the process reaches a coating value of 0.45 after $18 \mathrm{~min}$. After this time, sensor 1 shows no further increase of the coating mass. Thus, sensor 1 needs to be checked after the process regarding its function and position. The coating growth was monitored correctly for the other two sensors. All three sensors in this positioning allows for determination of the end point, in the case of one sensor failing.

\section{Process Monitoring}

The setup described in the "Setup for In-line Monitoring" and "In-line Monitoring of the Hot Melt Coating Process" sections indeed enables the in-line control of the hot melt coating process. The operator can follow the coating trajectory over time and out of specification occasions can be detected in real-time. An example of a frequently occurring event (in

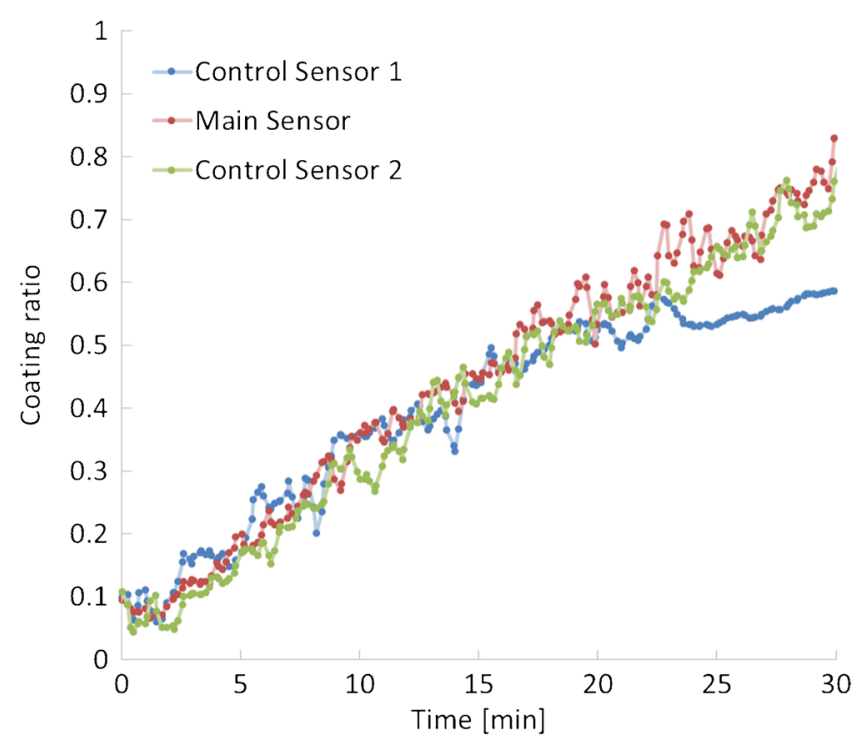

Fig. 13. Signals of the three sensors. Only two predicted the coating level correctly our setup) is the clogging of the tube, transporting the molten coating from the heating element to the spray nozzle. Corrective actions by the operator can be the elongation of the spraying time, or the increase in feed rate, after the tube has been cleaned.

An interesting example of a possibly faulty process is shown in Fig. 14. Here, the sensor did not notice the increase in coating over time, due to an unsuitable measurement position. At the process end, however, the powder bed developed enough height, to render the probe successful, and it indicated a regular process. In this questionable case, if the in-line monitoring had already been in place at that time, the process would have been stopped. Having only the usual end-control would have indicated a successful process nonetheless. In contrast, having full knowledge over time renders this batch out of specification. This is a warning, that the usual opinion of having more data being beneficial is only true if the additional data are of high quality and can be interpreted correctly.

\section{CONCLUSIONS}

This study demonstrates the advantages of using a multipoint near-infrared system for hot melt coating process monitoring. The CQA coating ratio was determined in different positions, and disturbances or inhomogeneities in the process chamber were promptly detected. Proper monitoring of the process and correct endpoint determination can be performed effectively with three sensors. In the case of a breakdown of one of these sensors, the quality of the batch and the endpoint can be determined with the remaining two sensors. Window fouling also has to be taken into consideration. Moreover, although particles in the fluid bed move very fast and often only void space was in front of the sensor, the appropriate quality of spectra was ensured due to the system's high acquisition speed.

Furthermore, an in-line-implementation was created that enabled the real-time supervision of the process, thus

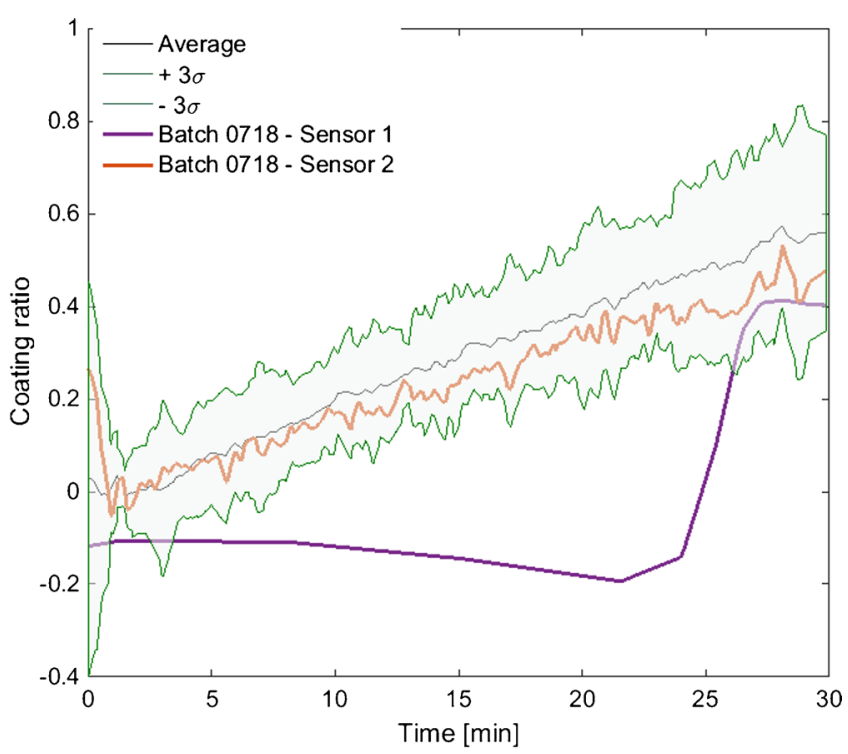

Fig. 14. Questionable case of a coating process with one sensor at an unsuitable measurement position. At the process end, a regular process is indicated 
facilitating the correct determination of the coating time and the fast detection of problems during the running process.

\section{ACKNOWLEDGEMENTS}

Open access funding provided by University of Graz. This work has been funded by the Austrian COMET Program under the auspices of the Austrian Federal Ministry of Transport, Innovation and Technology (bmvit); the Austrian Federal Ministry of Economy, Family and Youth (bmwfj); and the State of Styria (Styrian Funding Agency SFG). COMET is managed by the Austrian Research Promotion Agency FFG. The authors thank Manuel Zettl, Petra Hofstadler, and Moritz Gratzer for their help with operating the fluid bed.

Open Access This article is distributed under the terms of the Creative Commons Attribution 4.0 International License (http://creativecommons.org/licenses/by/4.0/), which permits unrestricted use, distribution, and reproduction in any medium, provided you give appropriate credit to the original author(s) and the source, provide a link to the Creative Commons license, and indicate if changes were made.

\section{REFERENCES}

1. Lee M-J, Seo D-Y, Lee H-E, Wang I-C, Kim W-S, Jeong M-Y, et al. In line NIR quantification of film thickness on pharmaceutical pellets during a fluid bed coating process. Int J Pharm. 2011;403(1-2):66-72. doi:10.1016/j.ijpharm.2010.10.022.

2. Wang LK, Heng PWS, Liew CV. Online monitoring of particle mass flow rate in bottom spray fluid bed coating - development and application. Int J Pharm. 2010;395(1-2):215-21. doi:10.1016/ j.ijpharm.2010.10.022.

3. Kirsch JD, Drennen JK. Determination of film-coated tablet parameters by near-infrared spectroscopy. J Pharm Biomed Anal. 1995;13(10):1273-81. doi:10.1016/0731-7085(95)01562-Y.

4. Cuq B, Gontard N, Guilbert S. Edible films and coatings as active layers. In: Rooney ML, editor. Active Food Packaging. New York: Springer US; 1995. p. 111-42.

5. Jannin V, Cuppok Y. Hot-melt coating with lipid excipients. Int $\mathbf{J}$ Pharm. 2013;457(2):480-7. doi:10.1016/j.ijpharm.2012.10.026.

6. Andersson M, Folestad S, Gottfries J, Johansson MO, Josefson M, Wahlund K-G. Quantitative analysis of film coating in a fluidized bed process by in-line NIR spectrometry and multivariate batch calibration. Anal Chem. 2000;72(9):2099-108. doi:10.1021/ac990256r.

7. Lee M-J, Park C-R, Kim A-Y, Kwon B-S, Bang K-H, Cho Y-S, et al. Dynamic calibration for the in-line NIR monitoring of film thickness of pharmaceutical tablets processed in a fluid-bed coater. J Pharm Sci. 2010;99(1):325-35. doi:10.1002/jps.21795.

8. Roggo Y, Jent N, Edmond A, Chalus P, Ulmschneider M. Characterizing process effects on pharmaceutical solid forms using near-infrared spectroscopy and infrared imaging. Eur J Pharm Biopharm. 2005;61(1-2):100-10. doi:10.1016/j.ejpb.2005.04.005.

9. Gendre C, Genty M, Boiret M, Julien M, Meunier L, Lecoq O, et al. Development of a Process Analytical Technology (PAT) for in-line monitoring of film thickness and mass of coating materials during a pan coating operation. Eur J Pharm Sci. 2011;43(4):244-50. doi:10.1016/j.ejps.2011.04.017.

10. Romero-Torres S, Pérez-Ramos JD, Morris KR, Grant ER. Raman spectroscopic measurement of tablet-to-tablet coating variability. J Pharm Biomed Anal. 2005;38(2):270-4. doi:10.1016/ j.jpba.2005.01.007.

11. Romero-Torres S, Pérez-Ramos JD, Morris KR, Grant ER. Raman spectroscopy for tablet coating thickness quantification and coating characterization in the presence of strong fluorescent interference. J Pharm Biomed Anal. 2006;41(3):811-9. doi:10.1016/ j.jpba.2006.01.033.
12. Kauffman JF, Dellibovi M, Cunningham CR. Raman spectroscopy of coated pharmaceutical tablets and physical models for multivariate calibration to tablet coating thickness. J Pharm Biomed Anal. 2007;43(1):39-48. doi:10.1016/j.jpba.2006.06.017.

13. Knop K, Kleinebudde P. PAT-tools for process control in pharmaceutical film coating applications. Int J Pharm. 2013;475:52736. doi:10.1016/j.ijpharm.2013.01.062.

14. Markl D, Zettl M, Hannesschläger G, Sacher S, Leitner M, Buchsbaum A, et al. Calibration-free in-line monitoring of pellet coating processes via optical coherence tomography. Chem Eng Sci. 2014;125:200-8. doi:10.1016/j.ces.2014.05.049.

15. Markl D, Hannesschläger G, Buchsbaum A, Sacher S, Khinast JG, Leitner M. In-line quality control of moving objects by means of spectral-domain OCT. Opt Lasers Eng. 2014;59:1-10. doi:10.1016/j.optlaseng.2014.02.008.

16. Markl D, Hannesschläger G, Sacher S, Leitner M, Khinast JG. Optical coherence tomography as a novel tool for in-line monitoring of a pharmaceutical film-coating process. Eur J Pharm Sci. 2014;55(1):58-67. doi:10.1016/j.ejps.2014.01.011.

17. Folttmann F, Knop K, Kleinebudde P, Pein M. In-line spatial filtering velocimetry for particle size and film thickness determination in fluidized-bed pellet coating processes. Eur J Pharm Biopharm. 2014;88(3):931-8. doi:10.1016/j.ejpb.2014.10.004.

18. Gendre C, Boiret M, Genty M, Chaminade P, Pean JM. Realtime predictions of drug release and end point detection of a coating operation by in-line near infrared measurements. Int $\mathrm{J}$ Pharm. 2011;421(2):237-43. doi:10.1016/j.ijpharm.2011.09.036.

19. Ciurczak EW, Igne B. Pharmaceutical and medical applications of near-infrared spectroscopy. 2nd ed. Boca Raton: CRC Press; 2015.

20. Frake P, Greenhalgh D, Grierson SM, Hempenstall JM, Rudd DR. Process control and end-point determination of a fluid bed granulation by application of near infra-red spectroscopy. Int J Pharm. 1997;151(1):75-80. doi:10.1016/S0378-5173(97)04894-1.

21. Scheibelhofer O, Balak N, Koller DM, Khinast JG. Spatially resolved monitoring of powder mixing processes via multiple NIR-probes. Powder Technol. 2013;243:161-70. doi:10.1016/ j.powtec.2013.03.035.

22. Scheibelhofer O, Balak N, Wahl PR, Koller DM, Glasser BJ, Khinast JG. Monitoring blending of pharmaceutical powders with multipoint NIR spectroscopy. AAPS PharmSciTech. 2013;14(1):234-44. doi:10.1208/s12249-012-9910-4.

23. Goode KR, Asteriadou K, Robbins PT, Fryer PJ. Fouling and cleaning studies in the food and beverage industry classified by cleaning type. Compr Rev Food Sci Food Saf. 2013;12(2):121-43. doi:10.1111/1541-4337.12000.

24. Martens H, Nielsen JP, Engelsen SB. Light scattering and light absorbance separated by extended multiplicative signal correction. Application to near-infrared transmission analysis of powder mixtures. Anal Chem. 2003;75(3):394-404. doi:10.1021/ ac020194w.

25. Scheibelhofer O, Hohl R, Salar-Behzadi S, Haack D, Koch K, Kerschhaggl P, et al. Automatic correction for window fouling of near infrared probes in fluidised systems. J Near Infrared Spectrosc. 2014;22(3):229-38. doi:10.1255/jnirs.1102.

26. Faustino JGM. Off-line dynamic image analysis vs PAT monitoring tool on a lab-scale hot melt coating process [master thesis]. Instituto Superior Técnico: Technical University of Lissabon; 2013.

27. Chiang LH, Leardi R, Pell RJ, Seasholtz MB. Industrial experiences with multivariate statistical analysis of batch process data. Chemom Intell Lab Syst. 2006;81(2):109-19. doi:10.1016/ j.chemolab.2005.10.006.

28. Kona R, Qu H, Mattes R, Jancsik B, Fahmy RM, Hoag SW. Application of in-line near infrared spectroscopy and multivariate batch modeling for process monitoring in fluid bed granulation. Int J Pharm Elsevier BV. 2013;452(1-2):63-72. doi:10.1016/ j.ijpharm.2013.04.039.

29. Kulcke A, Kerschhaggl P. High speed NIR spectral imaging in recycling applications. Proceedings of the 3rd international spectral imaging workshop. 2006 May 13; Graz, Austria. Graz: CTR; 2006. p. $40-7$.

30. Wold S, Kettaneh N, Fridén H, Holmberg A. Modelling and diagnostics of batch processes and analogous kinetic experiments. Chemom Intell Lab Syst. 1998;44(1-2):331-40. doi:10.1016/S01697439(98)00162-2. 